\title{
Hydrogen-Bonding Recognition-Induced Colorimetric Determination of Hydrazine Based on the Tryptophan Capped Gold Nanoparticles
}

\author{
Wenhua Gao,, ${ }^{1,2}$ Jing Xi, ${ }^{1}$ Yunsheng Chen, ${ }^{1}$ Song Xiao, ${ }^{3}$ Xingqing Wang, ${ }^{1}$ Jun Li, \\ Jincheng Xiao, ${ }^{1}$ and Yaowen Chen ${ }^{2}$ \\ ${ }^{1}$ Department of Chemistry, Shantou University, Guangdong, Shantou 515063, China \\ ${ }^{2}$ Analysis \& Testing Center, Shantou University, Guangdong, Shantou 515063, China \\ ${ }^{3}$ Department of Chemistry, Medical College of Guiyang, Guizhou, Guiyang 550004, China \\ Correspondence should be addressed to Wenhua Gao; whgao@stu.edu.cn
}

Received 27 June 2012; Accepted 4 September 2012

Academic Editor: Jairo Castillo-Chara

Copyright ( $\odot 2013$ Wenhua Gao et al. This is an open access article distributed under the Creative Commons Attribution License, which permits unrestricted use, distribution, and reproduction in any medium, provided the original work is properly cited.

A simple, cost-effective, and rapid colorimetric method for hydrazine detection using tryptophan-caped gold nanoparticles (TrpAuNPs) has been developed. Tryptophan (Trp) is a protein with reducibility and amino group which can reduce chloroauric acid $\left(\mathrm{HAuCl}_{4}\right)$ to AuNPs and modify the surface of AuNPs simultaneously. The Trp-AuNPs could be used to quantitatively detect hydrazine and showed different responses to vary concentration of hydrazine in an aqueous solution based on the aggregationinduced color change of Trp-AuNPs. The real water sample analysis verified the conclusion. The sensitivity of the detection system was influenced by the size of AuNPs which is determined by the $\mathrm{pH}$ of the detection system, the concentration of Trp, and the react time. We found that higher temperature contributed to more rapidly results. The detection system can detect as low as $1 \mu \mathrm{M}$ hydrazine. We expect our approach to have wide-ranging applications in the developing region for monitoring water quality in some areas.

\section{Introduction}

Hydrazine, a highly reactive base and a strong reducing agent, has been used as an important reactant in the preparation of pharmaceuticals, pesticides, photography chemicals, emulsifiers, and dyes in various chemical industries [1-3]. However, it is highly toxic and readily absorbed by oral, dermal, or inhalation routes of exposure, and long-term studies with laboratory animals indicate that hydrazine is mutagenic and carcinogenic [4]. Exposure to hydrazine at high levels (10 ppb, threshold limit value) can induce irritation of nose, temporary blindness, pulmonary edema, DNA damage, and even severe damage of the central nervous system [5-7]. In addition, contamination of rural drinking water supplies with hydrazine by livestock waste, organic wastes, and chemical fertilizers continues to be a problem. It has been a major concern throughout the world for several decades. Due to its widespread applications and toxic effects on humans, developing reliable and sensitive analytical methods for the selective detection of hydrazine is highly desirable.

So far, hydrazine can be routinely analyzed by a wide variety of techniques, such as gas chromatography [8], high performance liquid chromatography (HPLC) [9], ion chromatography [10], chemiluminescence (CL) [11], and electrochemical detection using a variety of chemically modified electrodes has also frequently been used [12]. Although these methods have high sensitivity, many of them are timeconsuming and labor-intensive due to the complex pretreatment, require expensive instrumentation and high cost of personnel, and are not readily adaptable to on-site, detection. Undoubtedly, it is of great significance to develop the simple, on-site and sensitive method for hydrazine detection.

In recent years, colorimetric methods have attracted much attention due to its low cost, simplicity, and practicality. Since color changes can be read out by the naked eye, colorimetric sensor does not require expensive or sophisticated 


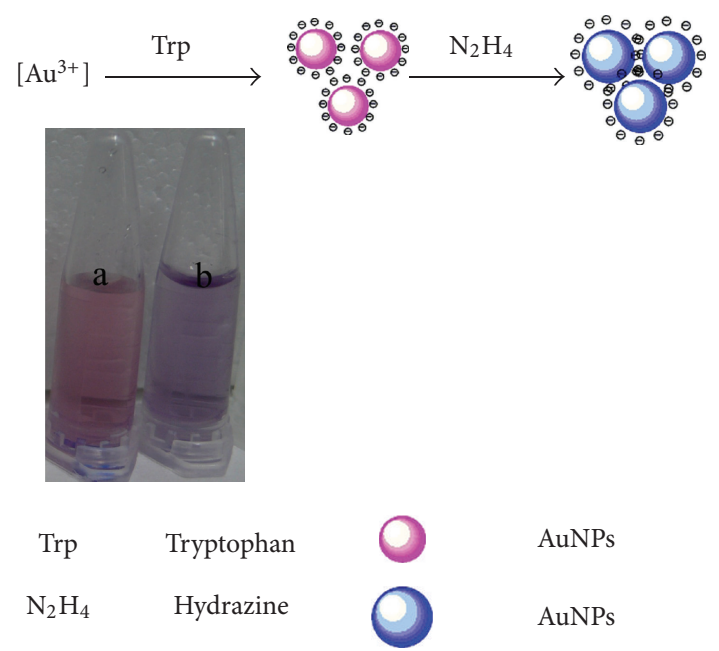

FIGURE 1: The schematic representation of the analytical process for detecting hydrazine. Insert: colorimetric visualization of the Trp-AuNPs generated in the absence of (a) and in the presence of hydrazine $\left(\mathrm{b} 2.0 \times 10^{-4}\right)$ in $\mathrm{pH} 5.0$ PBS.

instrumentation and may be applied to field analysis and point-of-care diagnosis. And these methods have shown great advantages over conventional assays, particularly in sensitivity, selectivity, and practicality.

For transformation of the detection events into color changes, a number of materials have been developed, including metal nanoparticles [13, 14], carbon nanotubes [15], grapheme oxide [16, 17], and conjugated polymers [18]. Among many materials, gold nanoparticle (AuNPs) has been regarded as the most promising candidate owing to their unique optical properties. Thus remarkable progress has been made on the design of AuNPs-based colorimetric biosensors owing to their intrinsically strong surface plasmon resonance (SPR) absorptions, with extremely high extinction coefficients, in the visible wavelength range. This color change effect is the result of the coupling of the SPR between particles in close proximity. Systems based on analyte induced aggregation of AuNPs have been employed for the colorimetric detection of heavy metal ions [19-21], melamine [22], acetamiprid [23], glucose [24], nitrate and nitrite [25], and some biological substances [26, 27].

These reported colorimetric assays generally require three steps: synthesis, modification of nanoparticles, and detection of targets. The aim of the former two steps is to fabricate a sensitive colorimetric probe for targets. And the functionalization of AuNPs is vital for triggering the colorimetric response of analytes, which also can be achieved during the synthesis of monodispersed AuNPs. Herein, we proposed a novel method to realize colorimetric detection of hydrazine based on tryptophan capped gold nanoparticles (Trp-AuNPs). In our study, there is only one step in the whole formation of Trp functionalized AuNPs without seeds. In this strategy, tryptophan served not only as a reductant but also a modifier that was capped AuNPs to avoid AuNPs aggregating autonomously to control the size of AuNPs. The colorimetric detection hydrazine was realized based on the visual color changing and ultraviolet visible (UV-Vis) absorption. In addition, the proposed method was successfully used for determination of the hydrazine in various environmental water samples. To the best of our knowledge, this is the first report to use this method for detecting hydrazine based on Trp caped AuNPs.

\section{Experimental}

2.1. Chemical Reagents. Chloroauric acid $\left(\mathrm{HAuCl}_{4}\right)$ and hydrazine were purchased from Sigma-Aldrich (St. Louis, MO, USA). Tryptophan (Trp) was purchased from Shanghai Biochemical Reagents Co. (Shanghai, China). Sodium dihydrogen phosphate and disodium hydrogen phosphate were purchased from Sinopharm Chemical Reagent Co. Ltd. (Shanghai, China). All other chemicals were of analytical reagent grade and used without further purification. And all stock solutions were prepared daily with double-deionized water obtained from a Milli-Q water purification system (Bedford, MA, USA).

2.2. Apparatus. UV-Vis spectra were obtained on a Lambda 950 spectrophotometer (Perkin Elmer, USA) at room temperature. The sample was thoroughly mixed with a shaker vortex (IKA Genius 3, Germany). The scanning electron microscope (SEM, JSM-6360LA, JEOL Ltd.) was also used to observe the aggregation and size distribution of Trp-AuNPs. A PHS-3CA precision $\mathrm{pH}$ meter (Dapu, China) was used in the experiment. The photo images of reaction solutions were recorded using a Coolpix 5400 digital color camera (Nikon, Tokyo, Japan).

2.3. Procedures. All glassware used in the following procedure were cleaned in a bath of freshly prepared aqua regia, rinsed thoroughly in double-deionized water, and dried in air prior to use. Before detecting hydrazine, $1.2 \mathrm{~mL}$ premixed solution in $1.5 \mathrm{~mL}$ centrifuge tube containing $600 \mu \mathrm{L} 2.0 \times$ $10^{-2} \mathrm{M}$ phosphate buffer solution (PBS, pH 5.0), $200 \mu \mathrm{L} 2.0$ $\times 10^{-3} \mathrm{M} \mathrm{HAuCl}_{4}$ and $400 \mu \mathrm{L} 1 \times 10^{-2} \mathrm{M}^{\mathrm{T}} \mathrm{Tr}$ were mixed for by a shaker vortex and then incubated in a $40^{\circ} \mathrm{C}$ water bath for $30 \mathrm{~min}$. Successively, $100 \mu \mathrm{L}$ of diverse concentrations of hydrazine were added to the premixed solution, shaking 20 times at room temperature. And then the relative UV-Vis absorbance spectra and photographs of the reaction solutions were recorded.

\section{Results and Discussion}

3.1. Principle of the Colorimetric Detection. Figure 1 described the principle of the colorimetric determination of hydrazine. First, Trp was used to reduce $\mathrm{HAuCl}_{4}$ to AuNPs. Due to electronic effect of the amino group of Trp, the formed AuNPs were capped by the Trp and could be monodisperse before the addition of hydrazine. Then, the Trp-AuNPs were unstable and tended to agglomerate gradually with addition of hydrazine, which is contributed to the increasing of inter-particles aggregation [28]. Meanwhile, the 


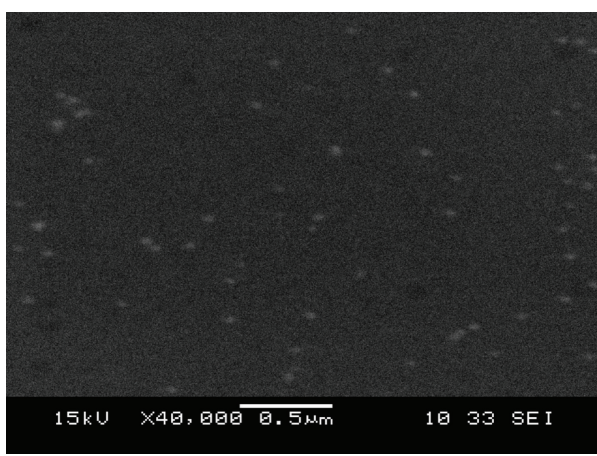

(a)

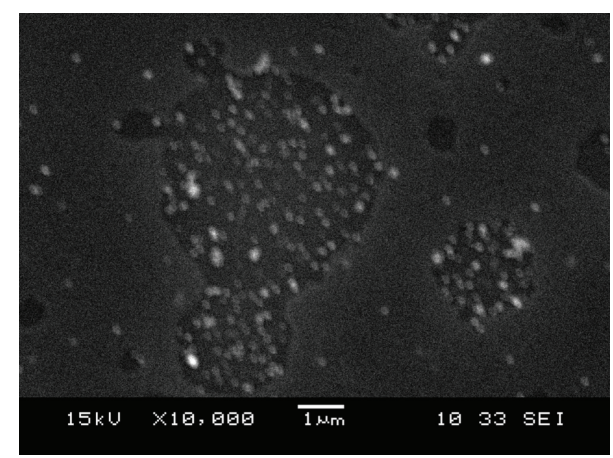

(b)

FIGURE 2: The SEM images of in the absence of (a) and in the presence of hydrazine (b). Condition: $1.2 \mathrm{~mL}$ premixed solution containing 1.0 $\times 10^{-2} \mathrm{M}$ PBS $(\mathrm{pH} 5.0), 3.1 \times 10^{-4} \mathrm{M} \mathrm{HAuCl}_{4}, 3.1 \times 10^{-3} \mathrm{M}$ Trp were mixed for by a shaker vortex and incubated in a $40^{\circ} \mathrm{C}$ water bath for $30 \mathrm{~min}$. Successively, $2.0 \times 10^{-4}$ of hydrazine were added to the premixed solution, shaking 20 times.

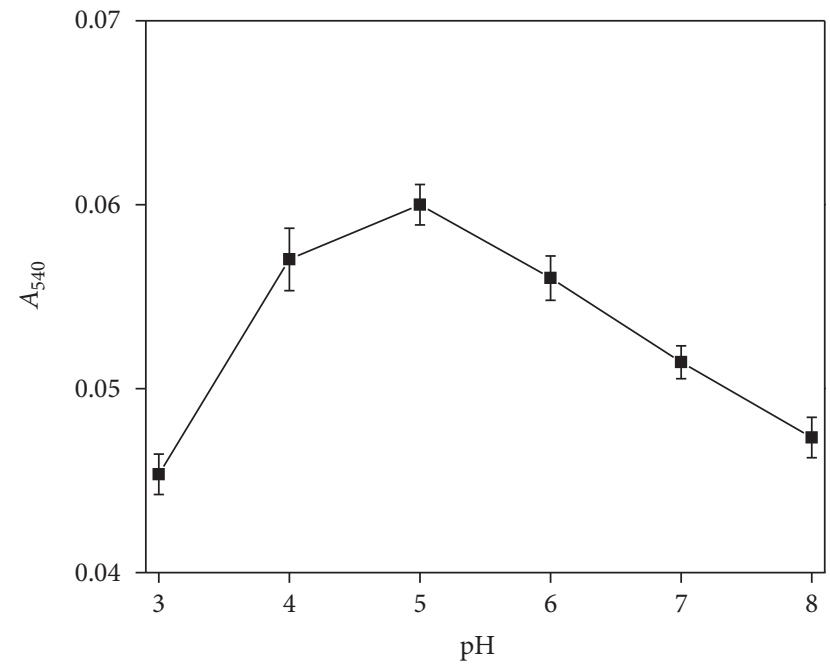

FIGURE 3: The effect of $\mathrm{pH}$. For conditions see Figure 2. Influence of the conditions in the addition of $7.57 \times 10^{-5} \mathrm{M}$ hydrazine.

color of reaction solution was changed from light purple red to purple (showed in the inset of Figure 1), which is result to the absorbance of reaction solution around $540 \mathrm{~nm}$ increased. Therefore, the concentration of hydrazine could be quantified by the absorption of $540 \mathrm{~nm}\left(\mathrm{~A}_{540}\right)$. In addition, a hydrazine molecule contained two amino groups, and Trp also had one amino group which meant that two such units could easily form extended arrays of hydrogen bonding $(\mathrm{NH} \cdots \mathrm{O}$ and $\mathrm{NH} \cdots \mathrm{N}$ ) which was particularly useful for sensing [29, 30]. Moreover, SEM was used to further verify the detection mechanism in Figure 2. Compared with monodisperse AuNPs (A), the interparticle distance of AuNPs decreased and resulted in obvious aggregations (B).

3.2. Optimum Conditions. In order to obtain the optimal condition, several impact factors were optimized. As well known, color change effect was the result of the coupling effect on the strong surface plasmon resonance (SPR) between particles in close proximity [31]. Thus we tested the $\mathrm{pH}$ of the reaction solution, the concentration of Trp, the temperature of the premixed solution (the premixed temperature) and the time of premixed solution (the premixed time).

3.2.1. Influence of $\mathrm{pH}$. The $\mathrm{pH}$ not only influenced the interaction between Trp-AuNPs and hydrazine, but also affected the stability of Trp-AuNPs. To investigate the effect of $\mathrm{pH}$ on the detection sensitivity, the reaction solutions at diverse $\mathrm{pH}$ values (3.0 to 7.0 in intervals of 1.0) were tested. Figure 3 shows that the relative UV-Vis absorption spectra in the presence of different values of $\mathrm{pH}$ (3.0 to 7.0) of $1.0 \times$ $10^{-2} \mathrm{M}$ PBS. The best sensitivity was obtained at $\mathrm{pH}$ 5.0. This was probably due to the fact that the hydrogen-bonding was quite weak in strong acidic solution. However, the ionization of Trp was adverse to the stability of Trp-AuNPs when $\mathrm{pH}$ was higher than 5. Therefore, $\mathrm{pH} 5.0$ was chosen for further experiments.

3.2.2. Influence of the Concentration of Trp. The selection of Trp both as a reductant and a modifier was due to its strong reducing capacity and amino group. The concentration of Trp was very important to the size and modification of TrpAuNPs, while the size of the Trp-AuNPs could affect the color and absorbance. Therefore, the concentration of Trp played a dominant role in the detection. Various concentrations of $\operatorname{Trp}$ (from 0 to $4.6 \times 10^{-3} \mathrm{M}$ ) were studied on the relevant optical absorption change. Figure 4 showed the UV-Vis absorption spectra in the presence of different concentrations of $\operatorname{Trp}(0$ to $4.6 \times 10^{-3} \mathrm{M}$ ) in $1 \times 10^{-2} \mathrm{M} \mathrm{PBS}(\mathrm{pH} 5.0)$. The experimental results showed that compared with others, $2.3 \times 10^{-3} \mathrm{M} \operatorname{Trp}$ was more sensitive in the detection. Thus, $2.3 \times 10^{-3} \mathrm{M} \operatorname{Trp}$ was selected for further experiments.

3.2.3. Influence of Operational Temperature. The operational temperature included the premixed temperature and binding temperature. Before adding the hydrazine, the Trp and $\mathrm{HAuCl}_{4}$ should be well mixed. Therefore, the premixed temperature was a key factor for the reaction between them. 


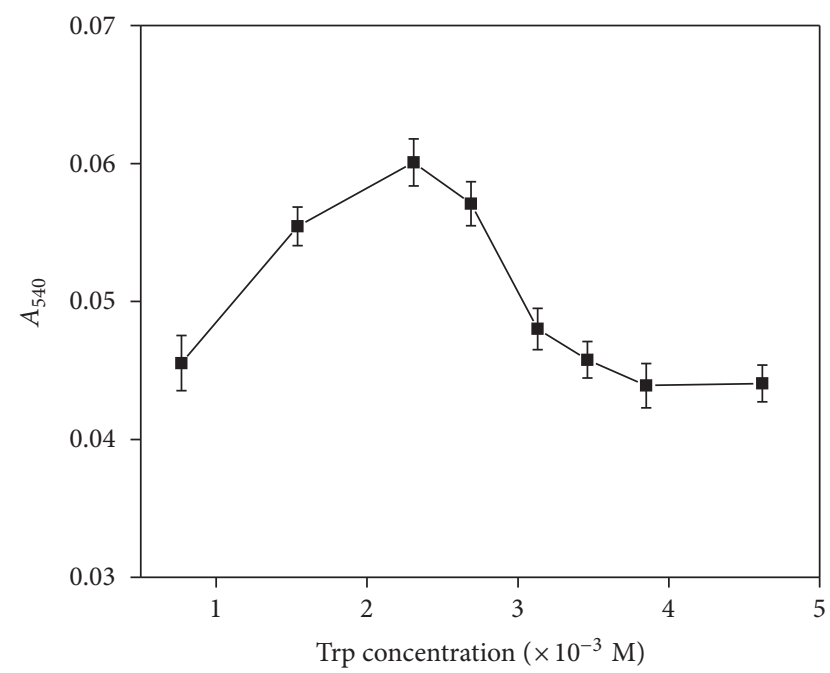

FIgURE 4: The effect of Trp concentration. For conditions see Figure 3.

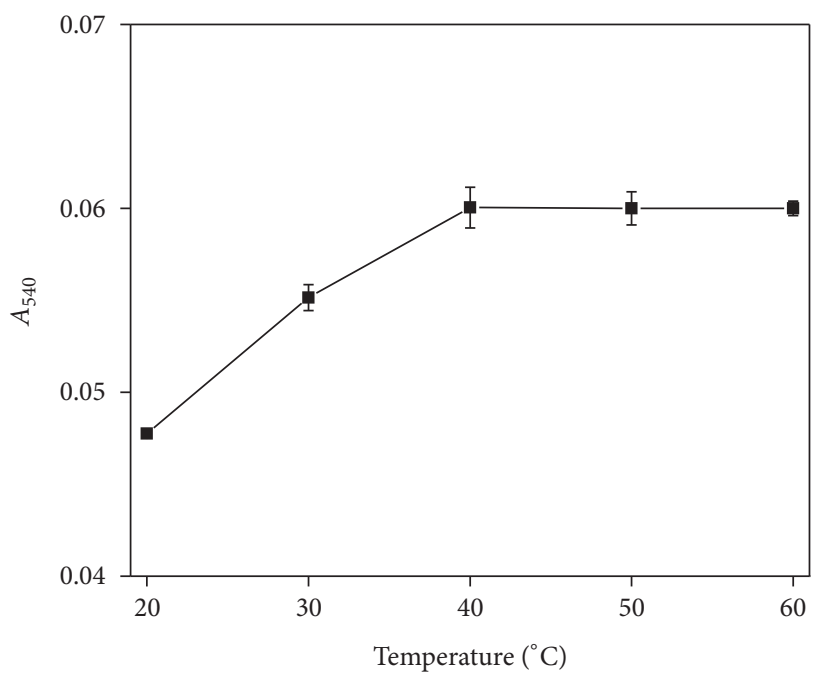

FIGURE 5: The effect of the premixed temperature. For conditions see Figure 3.

In particular, the mixture temperature also influenced the detection sensitivity. Thus, we tested in the range of $20-60^{\circ} \mathrm{C}$ at intervals of $10^{\circ} \mathrm{C}$. As shown in Figure 5, the absorption was the highest at $40^{\circ} \mathrm{C}$. The possible reasons were as follows: first, the high temperature was beneficial to the formation and modification of Trp-AuNPs. Second, the high temperature could induce self-aggregation of Trp-AuNPs, which was adverse to the assay [32]. Therefore, $40^{\circ} \mathrm{C}$ was chosen as the premixed temperature for all experiments.

When hydrazine added, the binding temperature affected hydrogen-bonding reaction. However, the self-aggregation of Trp-AuNPs attributing to high temperature should be avoided because the aggregation should be induced by hydrogen-bonding. Taking into account operational convenience, the room temperature was chosen as the binding temperature.

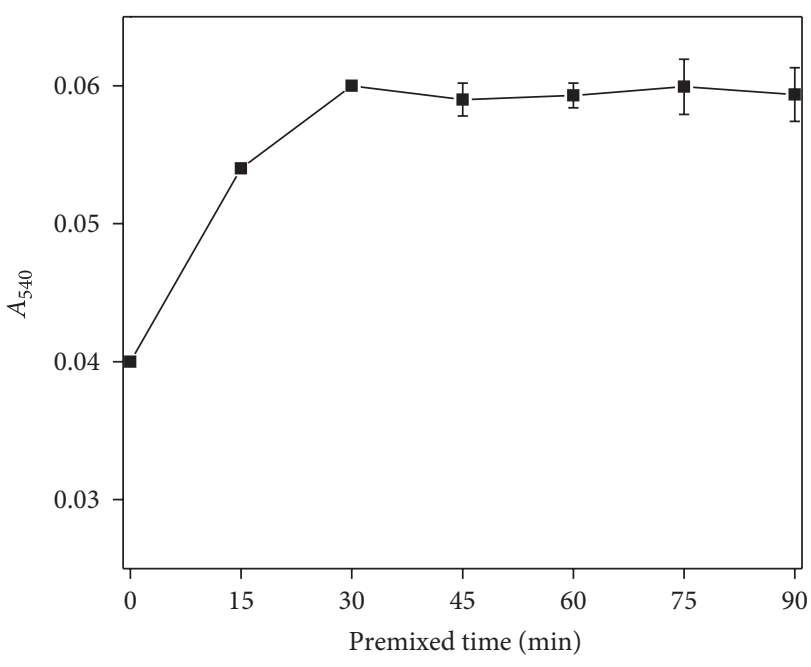

Figure 6: The effect of the premixed time. For conditions see Figure 3.

TABLE 1: The comparison of the LOD between different methods.

\begin{tabular}{|c|c|c|c|}
\hline Sample & Methods & $\begin{array}{c}\text { LOD } \\
\left(\times 10^{-6} \mathrm{M}\right)\end{array}$ & Reference \\
\hline Hydrazine & HPLC & 62.5 & [9] \\
\hline Hydrazine & Chemiluminescence & 0.5 & {$[11]$} \\
\hline Hydrazine & $\begin{array}{l}\text { Kinetic } \\
\text { spectrophotometric }\end{array}$ & 3.1 & [33] \\
\hline Hydrazine & Cyclic voltammetry & 11 & {$[34]$} \\
\hline Hydrazine & Amperometric assay & 8.5 & [35] \\
\hline Hydrazine & Colorimetry & 1.0 & $\begin{array}{c}\text { Current } \\
\text { work }\end{array}$ \\
\hline
\end{tabular}

3.2.4. Influence of Premixed Time. The premixed time also impacted the detection which was an inconvenient factor. To obtain the optimum condition, the mixture time was investigated in the range of 0-90 min at intervals of $15 \mathrm{~min}$. As shown in Figure 6, the value of $A_{540}$ sharpy increased at first $30 \mathrm{~min}$ and was kept in the same level after $30 \mathrm{~min}$. Therefore, $30 \mathrm{~min}$ was selected as the perfect premixed time.

\subsection{Colorimetric Detection of Hydrazine}

3.3.1. Sensitivity. Quantitative analysis was realized by the absorption of $540 \mathrm{~nm}\left(\mathrm{~A}_{540}\right)$. The corresponding UV-Vis absorption spectra were recorded. A linear correlation existed between the absorption ratio $\mathrm{A}_{540}$ and the hydrazine concentration in the range from $7.57 \times 10^{-6}$ to $2.01 \times 10^{-3} \mathrm{M}$ (shown in Figure 7). The regression equation was $Y=$ $(1.359 \pm 0.03982) \times 10^{-4}+(0.05166 \pm 0.0035) C(n=6)$ with a correlation coefficient (r) of 0.9957 , where $Y$ was the absorption at $540 \mathrm{~nm}$ and $C$ was hydrazine concentration $\left(\times 10^{-6} \mathrm{M}\right)$. The limit of detection (LOD) calculated as 3 times the standard deviation for the blank solution was $1.0 \times$ $10^{-6} \mathrm{M}$, which was significantly lower than the maximum hydrazine concentration allowed for water sources in China 
TABLE 2: Application of the proposed method to determination of hydrazine in real water samples spiked with different amounts of hydrazine.

\begin{tabular}{lccr}
\hline \multirow{2}{*}{ Sample } & \multicolumn{2}{c}{$\begin{array}{c}\text { Concentration of hydrazine hydrate }\left(\times 10^{-6} \mathrm{M}\right) \\
\text { Amount found }\end{array}$} & Recovery $(\%)$ \\
\hline \multirow{2}{*}{ Tap water } & 75.7 & $75.7 \pm 3.32$ & 100 \\
& 757 & $764 \pm 35.54$ & 101 \\
\hline \multirow{2}{*}{ Lake water } & 75.7 & $77.7 \pm 4.76$ & 103 \\
& 757 & $780 \pm 46.83$ & 103 \\
\hline \multirow{2}{*}{ Sea water } & 75.7 & $81.7 \pm 6.42$ & 108 \\
& 757 & $785 \pm 50.88$ & 104 \\
\hline
\end{tabular}

${ }^{a}$ Average value of three determinations \pm standard deviation.

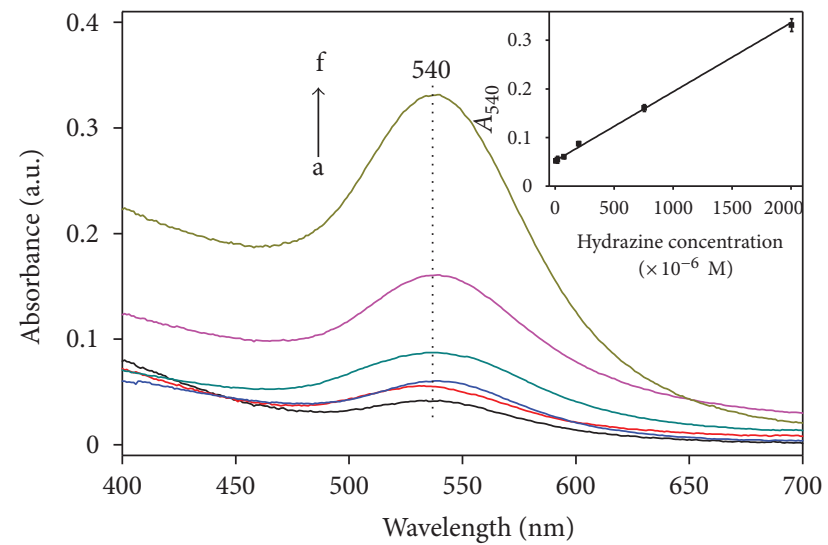

Figure 7: The relative UV-Vis absorbance spectra of reaction solution in the presence of different concentrations of hydrazine (the hydrazine concentration from a to $\mathrm{f}: 7.57 \times 10^{-6}, 2.01 \times 10^{-5}, 7.57 \times$ $10^{-5}, 2.01 \times 10^{-4}, 7.57 \times 10^{-4}$, and $2.01 \times 10^{-3} \mathrm{M}$ ) under the optimum conditions. Insert: the liner correlation between the absorption ratio $\mathrm{A}_{540}$ and the hydrazine concentration.

(6.25 × $10^{-6} \mathrm{M}$, Chinese National Standard GB 18061-2000). As shown in Table 1, the comparison of the LOD in different methods indicated that our study provided a better LOD than most of them. Moreover, our work provides a simpler method without any sophisticated instruments and complicated experiment operations.

What is more, to verify the rapidity and reliability of the proposed method, the relative UV-Vis spectra of the reaction solution were recorded at a regular interval of one minute in 10 min after adding two different concentration of hydrazine. The reaction time-dependent response curves were shown in Figure 8. The absorption ratio kept constant after $3 \mathrm{~min}$, indicating that the detection was extremely speedy and complete in three minute.

3.3.2. Selectivity. Various substances were most likely to be present in environmental water samples, such as metal ions, amino acids, and organics which could interfere with the determination of hydrazine. Therefore, the interferences in the 20 times concentration of hydrazine $\left(7.57 \times 10^{-5}\right)$ were used to carry out the experiment to investigate the selectivity of the proposed method. The values of $A_{540}$ were shown in

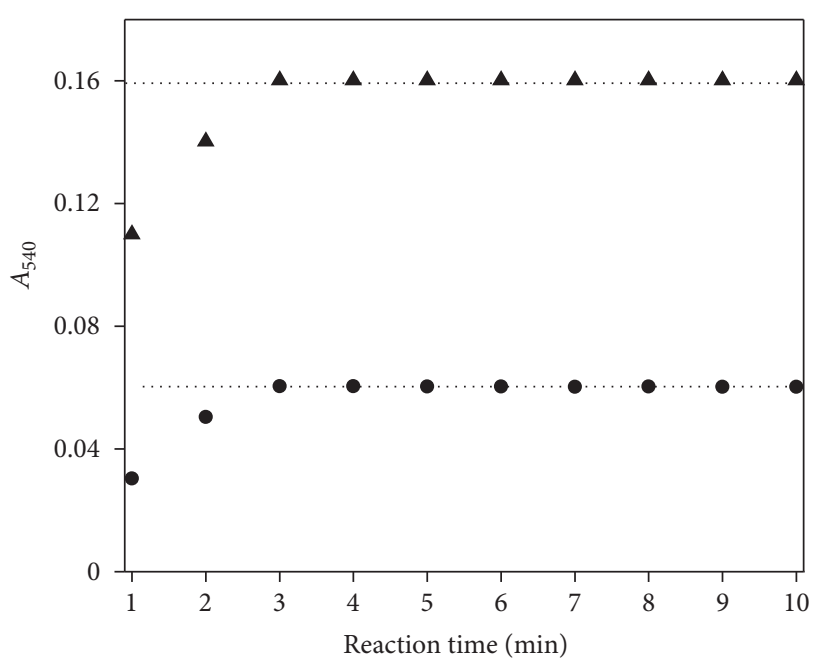

FIGURE 8: The reaction time in two different hydrazine concentration levels (a: $7.57 \times 10^{-5}$, b: $7.57 \times 10^{-4}$ ) under the optimum conditions.

Figure 9. Hydrazine obtained the largest absorption of $\mathrm{A}_{540}$, indicating that the determination of hydrazine had enough tolerable limit to the interferences.

3.4. Applications. In addition, we tested its colorimetric response to tap water samples, lake water samples, sea water samples, respectively, in order to evaluate the reliability of the proposed method. Samples of tap water were from our laboratory without any additional pretreatment. Lake water samples were from the artificial lake in our campus, and sea water samples were obtained from the shore of Shantou. The samples of lake water and sea water were diluted with double distilled water $(1: 1, \mathrm{v} / \mathrm{v})$. These real water samples were supplemented with two different concentrations of hydrazine, and the collected data of analytical recoveries and RSDs were shown in Table 2.

\section{Conclusions}

Based on the experimental results above, hydrazine could be detected with this simple, rapid, direct, and sensitive method, and it was suitable for routine analysis. The concentration of hydrazine in water samples can be determined by monitoring 


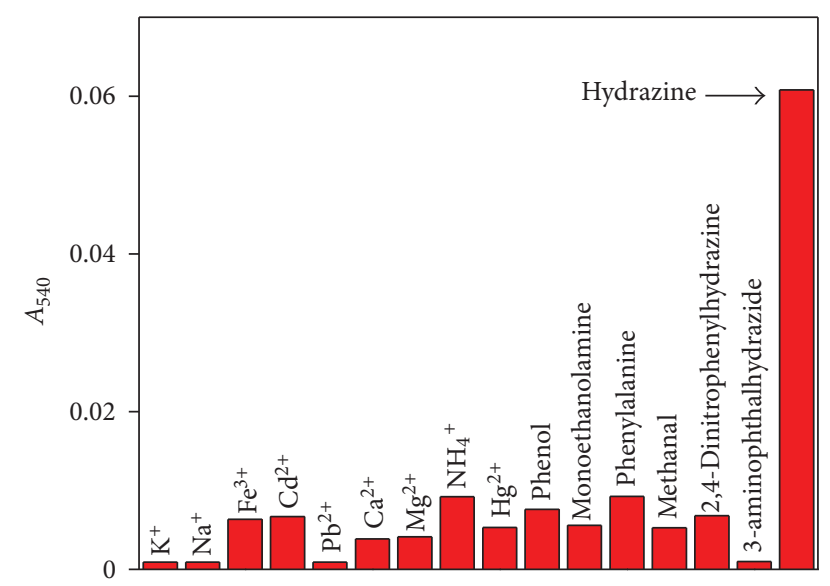

FIgURE 9: Absorption of reaction solution at $540 \mathrm{~nm}$ with the addition of hydrazine $\left(7.57 \times 10^{-5} \mathrm{M}\right)$ or other interferences under the optimum conditions.

with the naked eye or a UV-Vis spectrometer. The method showed relatively good selectivity for hydrazine over other hydrazinium with the lowest detection concentration of $1 \mu \mathrm{M}$. Especially, the merits made the proposed method specially useful for on-site screening hydrazine levels well below the current safety limit in drinking water.

\section{Acknowledgments}

The authors acknowledge financial support of this work by Research Start-up Funding of Shantou University (no. NTF10002), the Natural Science Foundation of Guangdong Province (no. S2011010005208), and Science and Technology Foundation of Guizhou Province (no. 20122026).

\section{References}

[1] A. Furst, R. C. Berlo, and S. Hooton, "Hydrazine as a reducing agent for organic compounds (catalytic hydrazine reductions)," Chemical Reviews, vol. 65, no. 1, pp. 51-68, 1965.

[2] A. Vass, J. Dudás, J. Tóth, and R. S. Varma, "Solvent-free reduction of aromatic nitro compounds with alumina-supported hydrazine under microwave irradiation," Tetrahedron Letters, vol. 42, no. 32, pp. 5347-5349, 2001.

[3] L. Cheng, W. X. Zhang, B. H. Ye, J. B. Lin, and X. M. Chen, "In situ solvothermal generation of 1,2,4-triazolates and related compounds from organonitrile and hydrazine hydrate: a mechanism study," Inorganic Chemistry, vol. 46, no. 4, pp. 1135-1143, 2007.

[4] M. Vogel, A. Büldt, and U. Karst, "Hydrazine reagents as derivatizing agents in environmental analysis-a critical review," Fresenius' Journal of Analytical Chemistry, vol. 366, no. 8, pp. 781-791, 2000.

[5] S. D. Zelnick, D. R. Mattie, and P. C. Stepaniak, "Occupational exposure to hydrazines: treatment of acute central nervous system toxicity," Aviation Space and Environmental Medicine, vol. 74, no. 12, pp. 1285-1291, 2003.

[6] S. Garrod, M. E. Bollard, A. W. Nicholls et al., "Integrated metabonomic analysis of the multiorgan effects of hydrazine toxicity in the rat," Chemical Research in Toxicology, vol. 18, no. 2, pp. 115-122, 2005.

[7] L. T. Ou and J. J. Street, "Hydrazine degradation and its effect on microbial activity in soil," Bulletin of Environmental Contamination and Toxicology, vol. 38, no. 1, pp. 179-183, 1987.

[8] J. A. Timbrell, J. M. Wright, and C. M. Smith, "Determination of hydrazine metabolites of isoniazid in human urine by gas chromatography," Journal of Chromatography, vol. 138, no. 1, pp. 165-172, 1977.

[9] H. M. Abdou, T. Medwick, and L. C. Bailey, "The determination of hydrazine and 1,1-dimethylhydrazine, separately or in mixtures, by high-pressure liquid chromatography," Analytica Chimica Acta, vol. 93, pp. 221-226, 1977.

[10] M. Mori, K. Tanaka, Q. Xu, M. Ikedo, H. Taoda, and W. $\mathrm{Hu}$, "Highly sensitive determination of hydrazine ion by ionexclusion chromatography with ion-exchange enhancement of conductivity detection," Journal of Chromatography A, vol. 1039, no. 1-2, pp. 135-139, 2004.

[11] A. Safavi and M. R. Baezzat, "Flow injection chemiluminescence determination of hydrazine," Analytica Chimica Acta, vol. 358, no. 2, pp. 121-125, 1998.

[12] B. K. Jena and C. Retna Raj, "Ultrasensitive nanostructured platform for the electrochemical sensing of hydrazine," Journal of Physical Chemistry C, vol. 111, no. 17, pp. 6228-6232, 2007.

[13] X. Xu, J. Wang, F. Yang, K. Jiao, and X. Yang, "Labelfree colorimetric detection of small molecules utilizing DNA oligonucleotides and silver nanoparticles," Small, vol. 5, no. 23, pp. 2669-2672, 2009.

[14] Z. Zhang, Z. Wang, X. Wang, and X. Yang, "Magnetic nanoparticle-linked colorimetric aptasensor for the detection of thrombin," Sensors and Actuators, B, vol. 147, no. 2, pp. 428-433, 2010.

[15] W. Shi, Q. Wang, Y. Long et al., "Carbon nanodots as peroxidase mimetics and their applications to glucose detection," Chemical Communications, vol. 47, no. 23, pp. 6695-6697, 2011.

[16] Y. L. Dong, H. G. Zhang, Z. U. Rahman et al., "Graphene oxide$\mathrm{Fe}_{3} \mathrm{O}_{4}$ magnetic nanocomposites with peroxidase-like activity for colorimetric detection of glucose," Nanoscale, vol. 4, pp. 3969-3976, 2012.

[17] F. Qu, T. Li, and M. Yang, "Colorimetric platform for visual detection of cancer biomarker based on intrinsic peroxidase activity of graphene oxide," Biosensors and Bioelectronics, vol. 26, no. 9, pp. 3927-3931, 2011.

[18] A. Asati, S. Santra, C. Kaittanis, S. Nath, and J. M. Perez, "Oxidase-like activity of polymer-coated cerium oxide nanopartieles," Angewandte Chemie, vol. 48, no. 13, pp. 2308-2312, 2009.

[19] K. W. Huang, C. J. Yu, and W. L. Tseng, "Sensitivity enhancement in the colorimetric detection of lead(II) ion using gallic acid-capped gold nanoparticles: improving size distribution and minimizing interparticle repulsion," Biosensors and Bioelectronics, vol. 25, no. 5, pp. 984-989, 2010.

[20] Y. R. Ma, H. Y. Niu, X. L. Zhang, and Y. Q. Cai, “Colorimetric detection of copper ions in tap water during the synthesis of silver/dopamine nanoparticles," Chemical Communications, vol. 47, pp. 12643-12645, 2011.

[21] C. C. Huang and H. T. Chang, "Parameters for selective colorimetric sensing of mercury(II) in aqueous solutions using mercaptopropionic acid-modified gold nanoparticles," Chemical Communications, no. 12, pp. 1215-1217, 2007. 
[22] Q. Cao, H. Zhao, Y. He et al., "Hydrogen-bonding-induced colorimetric detection of melamine by nonaggregation-based Au-NPs as a probe," Biosensors and Bioelectronics, vol. 25, no. 12, pp. 2680-2685, 2010.

[23] Q. Xu, S. Du, G. D. Jin, H. Li, and X. Y. Hu, "Determination of acetamiprid by a colorimetric method based on the aggregation of gold nanoparticles," Microchimica Acta, vol. 173, no. 3-4, pp. 323-329, 2011.

[24] C. Radhakumary and K. Sreenivasan, "Naked eye detection of glucose in urine using glucose oxidase immobilized gold nanoparticles," Analytical Chemistry, vol. 83, no. 7, pp. 2829-2833, 2011.

[25] W. L. Daniel, M. S. Han, J. S. Lee, and C. A. Mirkin, "Colorimetric Nitrite and Nitrate detection with gold nanoparticle probes and kinetic end points," Journal of the American Chemical Society, vol. 131, no. 18, pp. 6362-6363, 2009.

[26] B. I. Ipe, K. Yoosaf, and K. G. Thomas, "Functionalized gold nanoparticles as phosphorescent nanomaterials and sensors," Journal of the American Chemical Society, vol. 128, no. 6, pp. 1907-1913, 2006.

[27] S. Zhang, J. Wang, L. Han, C. Li, W. Wang, and Z. Yuan, "Colorimetric detection of bis-phosphorylated peptides using zinc(ii) dipicolylamine-appended gold nanoparticles," Sensors and Actuators, B, vol. 147, no. 2, pp. 687-690, 2010.

[28] W. Zhao, M. A. Brook, and Y. Li, "Design of gold nanoparticlebased colorimetric biosensing assays," ChemBioChem, vol. 9, no. 15, pp. 2363-2371, 2008.

[29] Z. L. Zhao, G. Zhang, Y. Gao, X. Y. Yang, and Y. H. Li, "A novel detection technique of hydrazine hydrate: modality change of hydrogen bonding-induced rapid and ultrasensitive colorimetric assay," Chemical Communications, vol. 47, pp. 12816-12818, 2011.

[30] K. Ai, Y. Liu, and L. Lu, "Hydrogen-bonding recognitioninduced color change of gold nanoparticles for visual detection of melamine in raw milk and infant formula," Journal of the American Chemical Society, vol. 131, no. 27, pp. 9496-9497, 2009.

[31] S. K. Ghosh and T. Pal, "Interparticle coupling effect on the surface plasmon resonance of gold nanoparticles: from theory to applications," Chemical Reviews, vol. 107, no. 11, pp. 4797-4862, 2007.

[32] L. Li, B. Li, D. Cheng, and L. Mao, "Visual detection of melamine in raw milk using gold nanoparticles as colorimetric probe," Food Chemistry, vol. 122, no. 3, pp. 895-900, 2010.

[33] S. Wang, L. Du, A. Zhang, and D. Liu, "Kinetic spectrophotometric determination of hydrazine with neutral red-nitrite system," Mikrochimica Acta, vol. 134, no. 3-4, pp. 167-170, 2000.

[34] K. I. Ozoemena and T. Nyokong, "Electrocatalytic oxidation and detection of hydrazine at gold electrode modified with iron phthalocyanine complex linked to mercaptopyridine selfassembled monolayer," Talanta, vol. 67, no. 1, pp. 162-168, 2005.

[35] J. S. Pinter, K. L. Brown, P. A. DeYoung, and G. F. Peaslee, "Amperometric detection of hydrazine by cyclic voltammetry and flow injection analysis using ruthenium modified glassy carbon electrodes," Talanta, vol. 71, no. 3, pp. 1219-1225, 2007. 

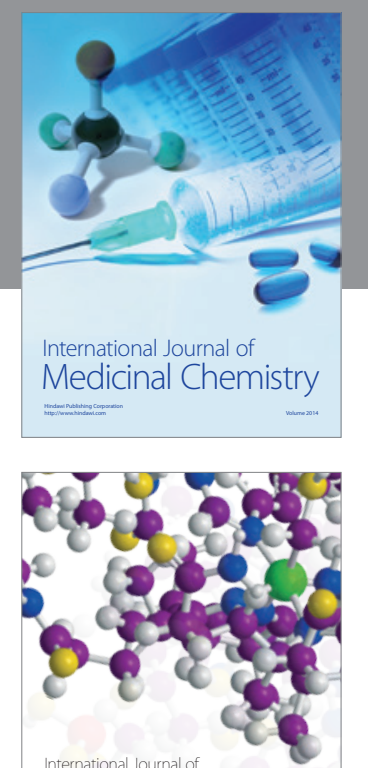

\section{Carbohydrate} Chemistry

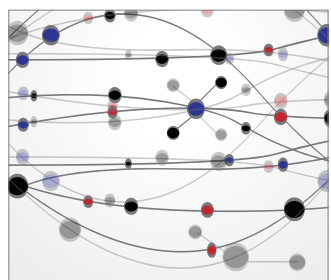

The Scientific World Journal
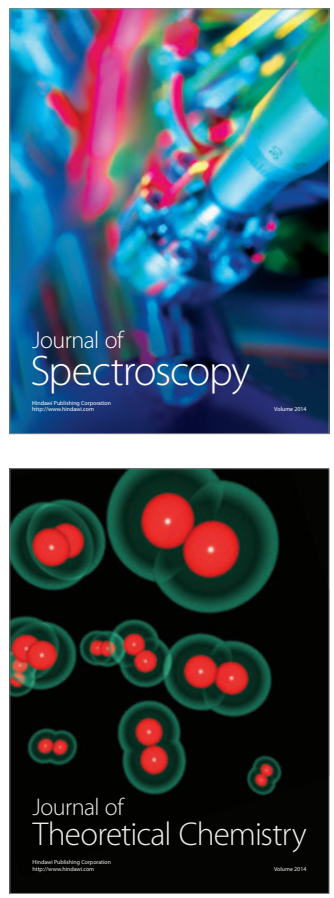
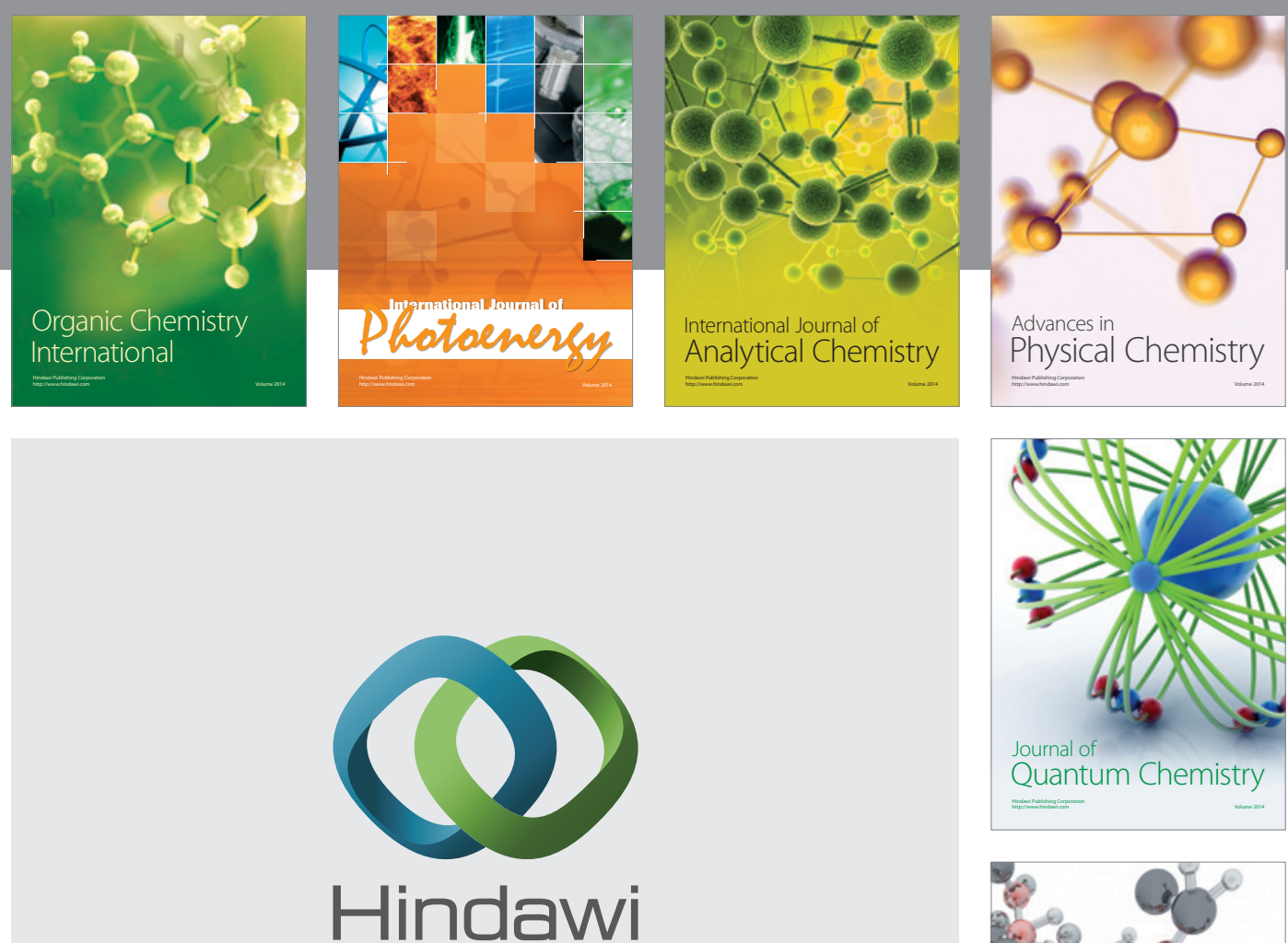

Submit your manuscripts at

http://www.hindawi.com

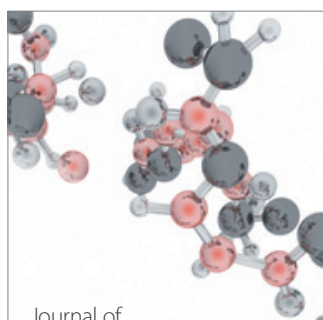

Analytical Methods

in Chemistry

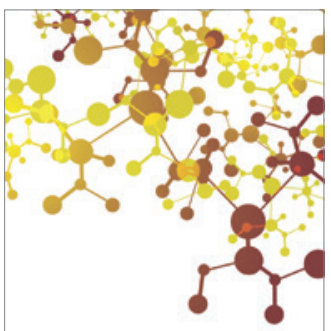

Journal of

Applied Chemistry

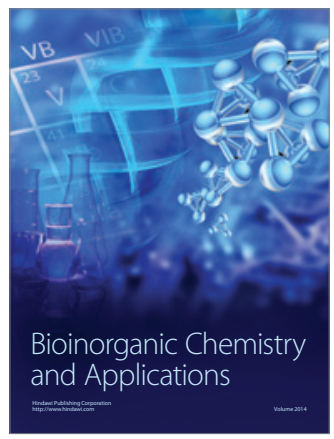

Inorganic Chemistry
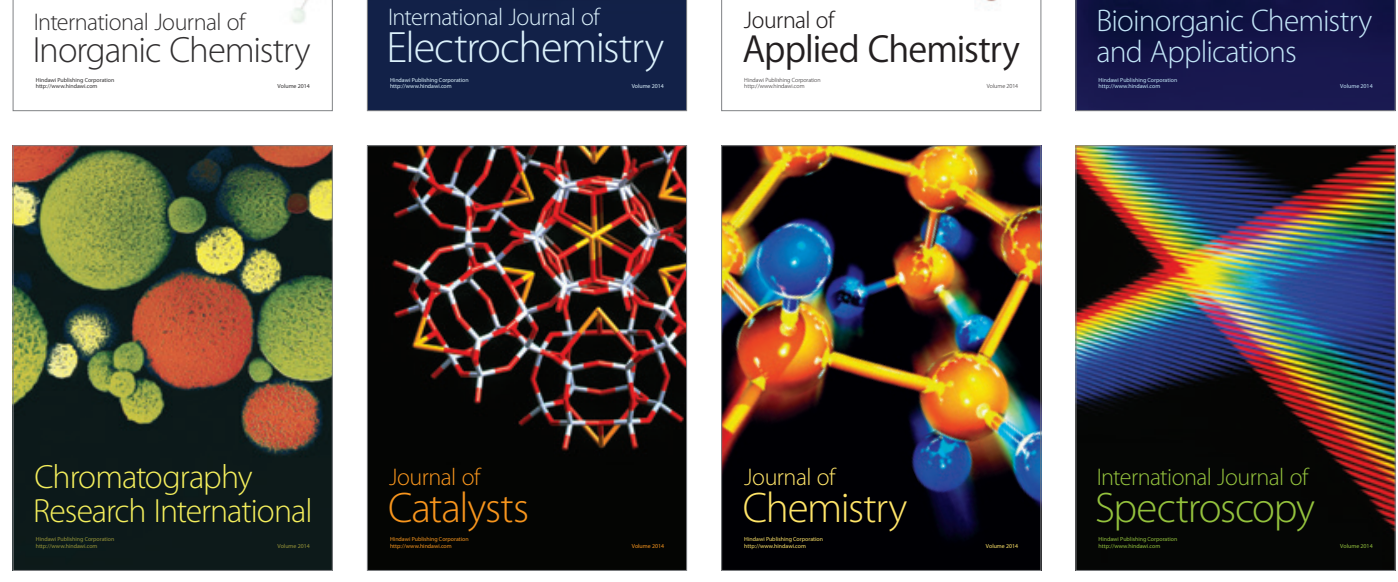